Article

\title{
The Particle as a Statistical Ensemble of Events in Stueckelberg-Horwitz-Piron Electrodynamics ${ }^{\dagger}$
}

\author{
Martin Land \\ Department of Computer Science, Hadassah Academic College, Jerusalem 91010, Israel; martin@hadassah.ac.il \\ + This paper is an extended version of our paper published in 3rd International Electronic and Flipped \\ Conference on Entropy and Its Applications, 1-10 November 2016.
}

Academic Editor: Antonio M. Scarfone

Received: 8 March 2017; Accepted: 17 May 2017; Published: 19 May 2017

\begin{abstract}
In classical Maxwell electrodynamics, charged particles following deterministic trajectories are described by currents that induce fields, mediating interactions with other particles. Statistical methods are used when needed to treat complex particle and/or field configurations. In Stueckelberg-Horwitz-Piron (SHP) electrodynamics, the classical trajectories are traced out dynamically, through the evolution of a $4 \mathrm{D}$ spacetime event $x^{\mu}(\tau)$ as $\tau$ grows monotonically. Stueckelberg proposed to formalize the distinction between coordinate time $x^{0}=c t$ (measured by laboratory clocks) and chronology $\tau$ (the temporal ordering of event occurrence) in order to describe antiparticles and resolve problems of irreversibility such as grandfather paradoxes. Consequently, in SHP theory, the elementary object is not a particle (a 4D curve in spacetime) but rather an event (a single point along the dynamically evolving curve). Following standard deterministic methods in classical relativistic field theory, one is led to Maxwell-like field equations that are $\tau$-dependent and sourced by a current that represents a statistical ensemble of instantaneous events distributed along the trajectory. The width $\lambda$ of this distribution defines a correlation time for the interactions and a mass spectrum for the photons emitted by particles. As $\lambda$ becomes very large, the photon mass goes to zero and the field equations become $\tau$-independent Maxwell's equations. Maxwell theory thus emerges as an equilibrium limit of SHP, in which $\lambda$ is larger than any other relevant time scale. Thus, statistical mechanics is a fundamental ingredient in SHP electrodynamics, and its insights are required to give meaning to the concept of a particle.
\end{abstract}

Keywords: classical gauge theory; pair creation/annihilation; temporal paradoxes

\section{Introduction}

In developing his interpretation of antiparticles as particles travelling backward in time, Stueckelberg [1,2] hoped to demonstrate that pair creation/annihilation processes would appear naturally in a thoroughly deterministic and classical relativistic Hamiltonian mechanics, once such a formalism could be properly constructed. In this picture, a particle is the worldline traced out by a spacetime event $x^{\mu}(\tau)$, for $\mu=0,1,2,3$, that evolves dynamically as the Poincaré invariant parameter proceeds monotonically from $\tau=-\infty$ to $\tau=\infty$. Stueckelberg argued that pair annihilation is observed when the coordinate time $x^{0}(\tau)$ reverses direction, because for some values $x_{2}^{0}>x_{1}^{0}$ on the laboratory clock there will be two solutions to $x^{0}(\tau)=x_{1}^{0}$ but no solution to $x^{0}(\tau)=x_{2}^{0}$. He proposed a covariant evolution equation of the form

$$
\frac{D}{D \tau} \dot{x}^{\mu}=\frac{d^{2} x^{\mu}}{d \tau^{2}}+\Gamma_{v \rho}^{\mu} \frac{d x^{v}}{d \tau} \frac{d x^{\rho}}{d \tau}=\frac{e}{M}\left[F^{\mu v}(x) g_{v \rho} \frac{d x^{\rho}}{d \tau}+G^{\mu}(x)\right]
$$


in which $\Gamma_{v \rho}^{\mu}$ is the Christoffel connection, $F^{\mu v}$ is the electromagnetic field tensor and the vector field $G^{\mu}(x)$ is required to overcome the mass-shell constraint

$$
\frac{D}{D \tau}\left(\frac{1}{2} M \dot{x}^{2}\right)=M \dot{x}_{\mu} \frac{D \dot{x}^{\mu}}{D \tau}=e \dot{x}_{\mu} G^{\mu}(x) \underset{G^{\mu} \rightarrow 0}{\longrightarrow} 0
$$

of standard relativity. By keeping $\dot{x}^{2}$ from changing sign, this constraint prevents the event from crossing the space-like region that separates future-oriented trajectories from past-oriented trajectories. However, Stueckelberg was not satisfied that he could justify a Hamiltonian $K$ that produces his evolution equation in flat spacetime from the unconstrained symplectic equations

$$
\frac{d x^{\mu}}{d \tau}=\dot{x}^{\mu}=\frac{\partial K}{\partial p_{\mu}} \quad \frac{d p^{\mu}}{d \tau}=\dot{p}^{\mu}=-\frac{\partial K}{\partial x_{\mu}}
$$

and instead continued his program in quantum mechanics, where, as in Feynman's spacetime diagrams, the event may tunnel probabilistically across the space-like region.

It should be noted that the Feynman-Stueckelberg interpretation of antiparticles relies on the Standard Model, in which all matter is composed of charged quarks and leptons subject to the strong and electroweak forces described by non-Abelian gauge theories. Thus, while the neutrino is electrically neutral, it carries the weak nuclear charge and is distinguished from the antineutrino by a time reversal produced through a generalization of (1) to the weak nuclear force. Moreover, neutral mesons are understood as quark bound states which are structurally symmetric under time reversal. Thus, under the electromagnetic force, the individual constituents of the $D 0$ meson (the bound state $u \bar{c}$ formed from an up quark and a charmed antiquark) may undergo time reversal separately, resulting in the $\overline{D 0}$ bound state $\bar{u} c$.

Horwitz and Piron [3] returned to some of these questions in constructing a canonical relativistic mechanics for the two-body problem. Introducing an invariant scalar interaction of the type

$$
K=\frac{p_{1}^{2}}{2 M_{1}}+\frac{p_{2}^{2}}{2 M_{2}}+V\left(\left|x_{1}-x_{2}\right|\right)
$$

and replacing

$$
R=\sqrt{\left(\mathbf{x}_{1}-\mathbf{x}_{2}\right)^{2}} \quad \longrightarrow \quad \rho=\sqrt{\left(\mathbf{x}_{1}-\mathbf{x}_{2}\right)^{2}-c^{2}\left(t_{1}-t_{2}\right)^{2}}
$$

in the argument of nonrelativistic scalar potentials, Horwitz et al. found solutions for relativistic generalizations of the standard central force problems, including quantum mechanical potential scattering and bound states [4-8]. Examination of radiative transitions [9-11] associated with these bound states suggests that the scalar interaction $V$ is required along with the four-vector potential $A^{\mu}$ in order to account for known phenomenology. Although the electromagnetic tensor interaction produced by $F^{\mu v}$ leaves individual particle masses invariant, the scalar interaction, which by way of (3) leads to the vector field $G^{\mu}=-\partial K / \partial x_{\mu}$ proposed by Stueckelberg, permits mass exchange in such transitions.

A related issue arises in defining the interaction picture in quantum field theory (QFT), required for the Dyson time-ordered perturbation expansion. On the one hand, the Haag theorem proves that any field obtained by unitary transformation of a free field must itself be a free field. On the other hand, the interacting fields of perturbation theory are defined by acting on free fields with a unitary transformation generated by an interaction Hamiltonian. To resolve this apparent contradiction, Seidewitz has observed [12] that Haag's proof relies on transformations generated by a Hamiltonian which is the 0-component of a four-vector and parameterized by $x^{0}$. He demonstrates that the construction of standard QFT in the Stueckelberg framework leads to an interaction picture obtained by acting on free fields with a unitary transformation generated by a scalar Stueckelberg Hamiltonian, as for example in Equation (11) below, and parameterized by $\tau$. Because this generator is invariant under 
Lorentz transformations, a crucial step in the Haag theorem is inapplicable, and the no-go result is averted. It is worth noting that even if the Stueckelberg Hamiltonian is $\tau$-independent, the interaction picture Hamiltonian will depend on $\tau$ and so permit mass exchange among particles and fields (just as a $t$-dependent nonrelativistic Hamiltonian permits nonconservation of energy).

Sa'ad, Horwitz, and Arshansky [13] found a more fundamental justification for the scalar field by studying the gauge invariance associated with (4). It has been shown [14] that the most general classical interaction consistent with the unconstrained quantum commutation relations

$$
\left[x^{\mu}, x^{v}\right]=0 \quad m\left[x^{\mu}, \dot{x}^{\nu}\right]=-i \hbar g^{\mu v}(x) \quad \mu, v=0,1,2,3
$$

is given by Stueckelberg's evolution Equation (1) with the substitutions

$$
F^{\mu v}(x) \rightarrow f^{\mu v}(x, \tau) \quad G^{\mu}(x) \rightarrow f^{5 \mu}(x, \tau),
$$

and that in flat Minkowski space, this system is equivalent to the Lagrangian

$$
L=\dot{x}^{\mu} p_{\mu}-K=\frac{1}{2} M \dot{x}^{\mu} \dot{x}_{\mu}+\frac{e}{c} \dot{x}^{\mu} a_{\mu}(x, \tau)+\frac{e}{c} \phi(x, \tau)
$$

where the electromagnetic field strength tensor

$$
f^{\mu v}=\partial^{\mu} a^{v}-\partial^{v} a^{\mu} \quad f^{5 \mu}=\partial_{\tau} a^{\mu}-\partial^{\mu} \phi
$$

is derived from five potentials.

This classical Lagrangian is unique up to the $\tau$-dependent gauge transformations

$$
a_{\mu}(x, \tau) \rightarrow a_{\mu}(x, \tau)+\partial_{\mu} \Lambda(x, \tau) \quad \phi(x, \tau) \rightarrow \phi(x, \tau)+\partial_{\tau} \Lambda(x, \tau)
$$

and the associated quantum mechanics

$$
i \hbar \partial_{\tau} \psi(x, \tau)=K \psi(x, \tau)=\left[\frac{1}{2 M}\left(p^{\mu}-\frac{e}{c} a^{\mu}\right)\left(p_{\mu}-\frac{e}{c} a_{\mu}\right)-\frac{e}{c} \phi\right] \psi(x, \tau)
$$

admits the additional invariance

$$
\psi(x, \tau) \rightarrow \exp \left[\frac{i e}{\hbar c} \Lambda(x, \tau)\right] \psi(x, \tau)
$$

when taken together with (10).

The generalization of Stueckelberg's framework to include $\tau$-dependent fields and gauge transformations thus succeeds in implementing his model of pair processes in classical mechanics, but also raises new questions. Perhaps most significantly, while defining the system in an unconstrained 8D phase space relaxes the a priori mass shell relation $\dot{x}^{2}=c^{2}$ and thus permits classical trajectories that reverse the direction of their time evolution, it also eliminates reparameterization invariance. This is because the mass shell constraint and reparameterization invariance are related features of a Lagrangian that is homogeneous of first degree in the velocities, which is not the case for (8). Moreover, in Stueckelberg-Horwitz-Piron (SHP) electrodynamics, the evolution parameter $\tau$ cannot be identified as the proper time of the motion, but is a dynamical quantity proportional to it through

$$
c^{2} d s^{2}(\tau)=-g_{\mu \nu} d x^{\mu} d x^{\nu}=-\dot{x}^{2}(\tau) d \tau^{2} \quad g_{\mu \nu}=\operatorname{diag}(-1,1,1,1)
$$

Therefore, the parameter $\tau$ plays the role of an irreducible chronological time, independent of the spacetime coordinates and similar to the external time $t$ in nonrelativistic Newtonian mechanics. It determines the temporal ordering of events, the order of their physical occurrence, which may 
be different from the order of observed coordinate times $x^{0}$ registered by laboratory clocks as the events appear in measuring apparatus. For example, in a classical, continuous version of a Feynman spacetime diagram, the event trajectory

$$
x(\tau)=(c t(\tau), \mathbf{x}(\tau))=\left(c\left(t_{0}+\dot{t}_{0} \tau-\frac{1}{2} \ddot{t}_{0} \tau^{2}\right), \mathbf{x}(\tau)\right)
$$

where $t_{0}, \dot{t}_{0}$ and $\ddot{t}_{0}$ are positive constants, reverses time direction at $\tau_{0}=\dot{t}_{0} / \ddot{t}_{0}$, and represents a pair annihilation process. Singling out three events in their $\tau$-chronological order of occurrence,

$$
\begin{array}{ccc}
\tau=0 & x^{0}=c t_{0} & \dot{x}^{0}=c \dot{t}_{0} \\
\tau=\tau_{0} & x^{0}=c\left(t_{0}+\dot{t}_{0}^{2} / 2 \ddot{t}_{0}\right) & \dot{x}^{0}=0 \\
\tau=2 \tau_{0} & x^{0}=c t_{0} & \dot{x}^{0}=-c \dot{t}_{0}
\end{array}
$$

the laboratory apparatus will first record both particle and antiparticle trajectories at $x^{0}=c t_{0}$, then the annihilation event at $x^{0}=c\left(t_{0}+\dot{t}_{0}^{2} / 2 \ddot{t}_{0}\right)$ and no particles for subsequent values of $x^{0}$. The classical antiparticle is identified here by its negative energy and no charge conjugation operation is necessary. Looking ahead to the current defined in (20), one sees that the electric charge $Q=\int d^{3} x j^{0}(x, \tau)$ will similarly reverse sign on this section of the trajectory. Although the laboratory apparatus observes the two events at $x^{0}=c t_{0}$ as simultaneous, they may be distinguished in SHP theory by other $\tau$-dependent interactions.

As Horwitz has observed, grandfather paradoxes may be resolved by noticing that the return trip to a past coordinate time $x^{0}$ must take place while the chronological time $\tau$ continues to increase. The occurrence of event $x^{\mu}\left(\tau_{1}\right)$ at $\tau_{1}$ is understood to be an irreversible process that cannot be changed by a subsequent event occurring at the same spacetime location, $x^{\mu}\left(\tau_{2}\right)=x^{\mu}\left(\tau_{1}\right)$ with $\tau_{2}>\tau_{1}$. This absence of closed time-like curves similarly applies in SHP quantum electrodynamics [15] where the particle propagator $G\left(x_{2}-x_{1}, \tau_{2}-\tau_{1}\right)$ vanishes unless $\tau_{2}>\tau_{1}$, thus preventing divergent matter loops, when $x_{2}=x_{1}$. This $\tau$-retarded causality was first shown by Feynman to be equivalent to the Feynman contour for propagators in connection with the path integral for the Klein-Gordon equation [16,17]. In SHP quantum electrodynamics (QED), it also emerges from the vacuum expectation value of $\tau$-ordered operator products. In the microscopic event dynamics described by this explicit distinction between chronological and coordinate time [18], a covariant Hamiltonian generates evolution of a $4 \mathrm{D}$ block universe defined at $\tau$ to an infinitesimally close $4 \mathrm{D}$ block universe defined at $\tau+d \tau$. Standard Maxwell electrodynamics emerges as an equilibrium limit in which the system becomes $\tau$-independent, and the $4 \mathrm{D}$ block universe remains static. The details of this $\tau$-dependence in the interacting fields and currents can be studied by reconciling classical SHP with classical Maxwell electromagnetic phenomenology.

\section{Classical SHP Electrodynamics}

In analogy with the notation $x^{0}=c t$, we adopt the formal designations

$$
x^{5}=c_{5} \tau \quad \partial_{5}=\frac{1}{c_{5}} \partial_{\tau} \quad j^{5}=c_{5} \rho \quad a_{5}=\frac{1}{c_{5}} \phi
$$

and the conventions

$$
\mu, v=0,1,2,3 \quad \alpha, \beta, \gamma=0,1,2,3,5 \quad g_{\alpha \beta}=\operatorname{diag}(-1,1,1,1, \pm 1)
$$


so that $c_{5} / c$ characterizes the relative rate of evolution in $\tau$, and as shown in Section 4 , SHP becomes Maxwell theory in the limit $c_{5} \rightarrow 0$. Writing (8) and (9) as

$$
L=\frac{1}{2} M \dot{x}^{\mu} \dot{x}_{\mu}+\frac{e}{c} \dot{x}^{\alpha} a_{\alpha} \quad f_{\beta}^{\alpha}=\partial^{\alpha} a_{\beta}-\partial_{\beta} a^{\alpha}
$$

the event dynamics are given by the Lorentz force

$$
M \ddot{x}^{\mu}=\frac{e}{c} f^{\mu}{ }_{\alpha}(x, \tau) \dot{x}^{\alpha} \quad \frac{d}{d \tau}\left(-\frac{1}{2} M \dot{x}^{2}\right)=g_{55} \frac{e c_{5}}{c} f^{5 \mu} \dot{x}_{\mu}
$$

equivalent to substituting the $\tau$-dependent fields (7) into Stueckelberg's evolution Equation (1) in flat spacetime. For the moment, we understand the factor $g_{55}$ as a choice of sign for the second of (19) and not a hint at some 5D metric structure.

To complete the dynamical picture, we re-express the velocity-potential interaction as a current-potential integral

$$
\begin{gathered}
\dot{X}^{\alpha} a_{\alpha} \rightarrow \int d^{4} x \dot{X}^{\alpha}(\tau) \delta^{4}(x-X(\tau)) a_{\alpha}(x, \tau)=\frac{1}{c} \int d^{4} x j^{\alpha}(x, \tau) a_{\alpha}(x, \tau) \\
j^{\alpha}(x, \tau)=c \dot{X}^{\alpha}(\tau) \delta^{4}(x-X(\tau))
\end{gathered}
$$

and choose some kinetic action term for the fields, the most obvious candidate being

$$
\mathcal{L}_{\text {kinetic }}=\frac{1}{4 c} f^{\alpha \beta}(x, \tau) f_{\alpha \beta}(x, \tau)
$$

which generalizes to 5D the standard Maxwell action [19]. Combining (20) and (21), the electromagnetic action

$$
S_{\mathrm{em}}=\int d^{4} x d \tau\left\{\frac{e}{c^{2}} j^{\alpha}(x, \tau) a_{\alpha}(x, \tau)-\frac{1}{4 c} f^{\alpha \beta}(x, \tau) f_{\alpha \beta}(x, \tau)\right\}
$$

can be varied with respect to $a_{\alpha}$ to produce Maxwell-like field equations, admitting a wave equation with associated Green's function whose solutions describe the fields induced by specific event trajectories. However, one is immediately confronted by conceptual difficulties in attempting to describe even the simple case of low energy Coulomb scattering. First, although the current $j^{\alpha}(x, \tau)$ and the potential $a_{\alpha}(x, \tau)$ are individually constructed to be vector + scalar representations of $\mathrm{O}(3,1)$ on physical grounds, the 5D scalar structure of the electromagnetic action (22) places all five components on the same algebraic footing. This suggests an underlying formal symmetry larger than $O(3,1)$ but containing it as a subgroup: $\mathrm{O}(4,1)$ for the choice $g_{55}=1$ or $\mathrm{O}(3,2)$ symmetry for $g_{55}=-1$. The formal 5D scalar structure survives in the wave equation and these symmetry considerations cannot be entirely ignored. Second, because $x^{\mu}$ and $\tau$ are introduced to play very different roles, the rest frame of an event is $\tau$-dependent. Thus, a "static" particle-an event evolving uniformly along the $x^{0}$ axis in its rest frame-is described by $x_{\text {rest }}=(c \tau, 0)$. It turns out that the potential at an observation point $x=(c t, \mathbf{x})$ induced by this particle is of the form $\delta\left((\tau-t)^{2}-|\mathbf{x}|^{2} / c^{2}\right)$ with support sharply focused on the lightcone of the source event's immediate location. A test event evolving uniformly as $x_{\text {test }}=\left(c\left(\tau+t_{0}\right), \mathbf{R}\right)$ will experience the potential $\delta\left(t_{0}^{2}-|\mathbf{R}|^{2} / c^{2}\right)$ for all $\tau$, rendering comparison with experiment nearly impossible. It is worth noting that the problem of clock synchronization between the source and test events (characterized here by $x_{\text {test }}^{0}(0)=c t_{0}$ ) is not a problem for the quantized theory where the use of asymptotic states with sharp mass implies maximum uncertainty in the location $x^{\mu}$ of the event in $\tau$. By introducing a degree of uncertainty into the definition of the classical electromagnetic action, a reasonable theory may be constructed. 


\section{Non-Local Field Kinetics $\leftrightarrow$ Ensemble of Events}

The problem of potentials with $\delta$-function support can be repaired by writing the action with a slightly less obvious candidate for the field kinetic term, the non-local form

$$
S_{\mathrm{em}}=\int d^{4} x d \tau\left\{\frac{e}{c^{2}} j^{\alpha}(x, \tau) a_{\alpha}(x, \tau)-\int \frac{d s}{\lambda} \frac{1}{4 c}\left[f^{\alpha \beta}(x, \tau) \Phi(\tau-s) f_{\alpha \beta}(x, s)\right]\right\}
$$

where $\lambda$ is a parameter with dimensions of time. The field interaction kernel is

$$
\Phi(\tau)=\delta(\tau)-(\xi \lambda)^{2} \delta^{\prime \prime}(\tau)=\int \frac{d \kappa}{2 \pi}\left[1+(\xi \lambda \kappa)^{2}\right] e^{-i \kappa \tau}
$$

where

$$
\xi=\frac{1}{2}\left[1+\left(\frac{c_{5}}{c}\right)^{2}\right]
$$

is chosen so that the low energy Lorentz force agrees with Coulomb's law. The kinetic term now includes $\left(\partial_{\tau} f^{\alpha \beta}(x, \tau)\right)\left(\partial_{\tau} f_{\alpha \beta}(x, \tau)\right)$ which explicitly breaks the $5 \mathrm{D}$ symmetry to $\mathrm{O}(3,1)$ while maintaining gauge symmetry. We write the inverse function of the interaction kernel as

$$
\varphi(\tau)=\lambda \Phi^{-1}(\tau)=\lambda \int \frac{d \kappa}{2 \pi} \frac{e^{-i \kappa \tau}}{1+(\xi \lambda \kappa)^{2}}=\frac{1}{2 \xi} e^{-|\tau| / \xi \lambda}
$$

which satisfies

$$
\int \frac{d s}{\lambda} \varphi(\tau-s) \Phi(s)=\delta(\tau) \quad \int \frac{d \tau}{\lambda} \varphi(\tau)=1 .
$$

Varying the action (23) with respect to the potentials, leads to field equations

$$
\partial_{\beta} f_{\Phi}^{\alpha \beta}(x, \tau)=\partial_{\beta} \int \frac{d s}{\lambda} \Phi(\tau-s) f^{\alpha \beta}(x, s)=\frac{e}{c} j^{\alpha}(x, \tau)
$$

describing the non-local superposition of fields $f_{\Phi}^{\alpha \beta}$ sourced by the instantaneous event current $j^{\alpha}(x, \tau)$. Using (27) to remove $\Phi(\tau)$ from the LHS and writing the Bianchi identity, we obtain equations for the local field sourced by a non-local superposition of event currents,

$$
\begin{aligned}
& \partial_{\beta} f^{\alpha \beta}(x, \tau)=\frac{e}{c} \int d s \varphi(\tau-s) j^{\alpha}(x, s)=\frac{e}{c} j_{\varphi}^{\alpha}(x, \tau) \\
& \partial_{\alpha} f_{\beta \gamma}+\partial_{\gamma} f_{\alpha \beta}+\partial_{\beta} f_{\gamma \alpha}=0
\end{aligned}
$$

which are formally similar to Maxwell's equations in 5D and are called pre-Maxwell equations. Rewriting the field equations in $4 \mathrm{D}$ tensor, vector and scalar components, they take the form

$$
\begin{array}{ll}
\partial_{\nu} f^{\mu \nu}-\frac{1}{c_{5}} \frac{\partial}{\partial \tau} f^{5 \mu}=\frac{e}{c} j_{\varphi}^{\mu} & \partial_{\mu} f^{5 \mu}=\frac{e}{c} j_{\varphi}^{5}=\frac{c_{5}}{c} e \rho_{\varphi} \\
\partial_{\mu} f_{v \sigma}+\partial_{\nu} f_{\sigma \mu}+\partial_{\sigma} f_{\mu \nu}=0 & \partial_{\nu} f_{5 \mu}-\partial_{\mu} f_{5 v}+\frac{1}{c_{5}} \frac{\partial}{\partial \tau} f_{\mu v}=0
\end{array}
$$

which may be compared with the 3-vector form of Maxwell's equations

$$
\begin{array}{ll}
\nabla \times \mathbf{B}-\frac{1}{c} \frac{\partial}{\partial t} \mathbf{E}=\frac{e}{c} \mathbf{J} & \nabla \cdot \mathbf{E}=\frac{e}{c} J^{0} \\
\nabla \cdot \mathbf{B}=0 & \nabla \times \mathbf{E}+\frac{1}{c} \frac{\partial}{\partial t} \mathbf{B}=0
\end{array}
$$


showing that $f^{5 \mu}$ plays the role of the vector electric field and $f^{\mu v}$ plays the role of the magnetic field. It follows from (29) that current conservation takes the form

$$
\partial_{\alpha} j^{\alpha}=\partial_{\mu} j^{\mu}(x, \tau)+\frac{1}{c_{5}} \partial_{\tau} j^{5}(x, \tau)=0
$$

so that a change in the divergence in the $4 \mathrm{D}$ Maxwell-like current $j^{\mu}$ must be compensated by the addition or subtraction of events through $j^{5}$. The fifth component of the current $j^{5}(x, \tau)=c_{5} \rho(x, \tau)$ thus plays the role of an event density, the probability that a material event occurs at the spacetime point $x$ at the chronological time $\tau$. Integrated over all spacetime,

$$
\frac{d}{d \tau} \int d^{4} x \rho(x, \tau)=-\int d^{4} x \partial_{\mu} j^{\mu}(x, \tau)=0
$$

expresses the conservation of total event number.

Rewriting the source of the inhomogeneous Equation (29) as

$$
j_{\varphi}^{\alpha}(x, \tau)=\int d s \varphi(\tau-s) j^{\alpha}(x, s)=\frac{1}{2 \xi} \int d s e^{-|s| / \xi \lambda} j^{\alpha}(x, \tau-s)
$$

we recognize $j_{\varphi}^{\alpha}(x, \tau)$ as a weighted superposition of currents, each originating at an event $X^{\mu}(\tau-s)$ displaced from $X^{\mu}(\tau)$ by an amount $s$ along the worldline. It is useful to regard this superposition as the current produced by an ensemble of events in the neighborhood of $X^{\mu}(\tau)$, a view encouraged by the particular weight function $\varphi(s)$. Given a Poisson distribution describing the occurrence of independent random events with a constant average rate of $1 / \lambda \xi$ events per second, the average time between events is $\lambda \xi$ and the probability at $\tau$ that the next event will occur following a time interval $s>0$ is just $\varphi(s) / \lambda=e^{-s / \xi \lambda} / \xi \lambda$. Extending the displacement to positive and negative values, the ensemble is constructed by assembling a set of instantaneous event currents $j^{\alpha}(x, \tau-s)$ along the worldline, each weighted by $\varphi(s)$, the probability that the occurrence of this event is delayed from $\tau$ by an interval of at least $|s|$. We will see that the leading term of the Green's function manifestly breaks $5 \mathrm{D}$ symmetry to $\mathrm{O}(3,1)$ and the causality relations embedded in this term select the one event from this ensemble for which the interacting events are at light-like separation, depending on their relative $\tau$-synchronization.

The ensemble can also be understood by thinking of $j_{\varphi}^{\alpha}(x, \tau)$ as a random variable describing the probability of finding a current density at $x$ at a given $\tau$. Then we may consider a correlation function for the event density of the type

$$
\langle\rho(\tau) \rho(s)\rangle=\frac{1}{\mathcal{N}} \int d^{4} x \rho(x, \tau) \rho(x, s)
$$

where $\mathcal{N}$ is a normalization. For a uniformly moving event with $X^{\mu}(\tau)=u^{\mu} \tau$, the raw event current (20) leads to

$$
\langle\rho(\tau) \rho(s)\rangle=\frac{c^{2}}{\mathcal{N}} \int d^{4} x \delta^{4}(x-u \tau) \delta^{4}(x-u s)=\frac{c^{2} \delta^{3}(\mathbf{0})}{\left|u^{0}\right| \mathcal{N}} \delta(\tau-s)
$$

showing that the currents at differing times $\tau \neq s$ are uncorrelated. For the ensemble current defined in (29), the correlation becomes

$$
\begin{aligned}
\left\langle\rho_{\varphi}(\tau) \rho_{\varphi}(s)\right\rangle & =\frac{c^{2}}{\mathcal{N}} \int d \tau^{\prime} d s^{\prime} d^{4} x \varphi\left(\tau-\tau^{\prime}\right) \varphi\left(s-s^{\prime}\right) \delta^{4}\left(x-u \tau^{\prime}\right) \delta^{4}\left(x-u s^{\prime}\right) \\
& =\frac{c^{2} \delta^{3}(\mathbf{0})}{\left|u^{0}\right| \mathcal{N}} \int d \tau^{\prime} \varphi\left(\tau-\tau^{\prime}\right) \varphi\left(\tau^{\prime}-s\right) \\
& =\frac{c^{2} \delta^{3}(\mathbf{0})}{4 \xi^{2}\left|u^{0}\right| \mathcal{N}} \int d \tau^{\prime} e^{-\left|\tau-\tau^{\prime}\right| / \zeta^{\prime} \lambda-\left|\tau^{\prime}-s\right| / \xi \lambda}
\end{aligned}
$$


so that taking $\tau>s$ and separating the integral into three intervals punctuated by $s$ and $\tau$ leads to

$$
\left\langle\rho_{\varphi}(\tau) \rho_{\varphi}(s)\right\rangle=\frac{\lambda c^{2} \delta^{3}(\mathbf{0})}{4 \xi\left|u^{0}\right| \mathcal{N}}\left(1+\frac{\tau-s}{\xi \lambda}\right) e^{-(\tau-s) / \xi \lambda}
$$

with a time dependence characteristic of an Ornstein-Uhlenbeck process. Regarding the smoothed current $j_{\varphi}^{\alpha}(x, \tau)$ produced by an event $X^{\mu}(\tau)$ as the instantaneous current produced by an ensemble of events, this correlation suggests that the ensemble is the result of the Brownian motion found by subjecting $X^{\mu}(\tau)$ to a random force under viscous drag.

The pre-Maxwell equations in Lorenz gauge lead to the wave equation

$$
\partial_{\beta} \partial^{\beta} a^{\alpha}=\left(\partial_{\mu} \partial^{\mu}+\partial_{\tau} \partial^{\tau}\right) a^{\alpha}=\left(\partial_{\mu} \partial^{\mu}+\frac{g_{55}}{c_{5}^{2}} \partial_{\tau}^{2}\right) a^{\alpha}=-\frac{e}{c} j_{\varphi}^{\alpha}(x, \tau)
$$

whose solutions may respect $5 \mathrm{D}$ symmetries broken by the $\mathrm{O}(3,1)$ symmetry of the event dynamics. A Green's function solution to

$$
\left(\partial_{\mu} \partial^{\mu}+\frac{g_{55}}{c_{5}^{2}} \partial_{\tau}^{2}\right) G(x, \tau)=-\delta^{4}(x) \delta(\tau)
$$

can be used to obtain potentials in the form

$$
a^{\alpha}(x, \tau)=-\frac{e}{c} \int d^{4} x^{\prime} d \tau^{\prime} G\left(x-x^{\prime}, \tau-\tau^{\prime}\right) j_{\varphi}^{\alpha}\left(x^{\prime}, \tau^{\prime}\right) .
$$

The principal part Green's function [20] is

$$
\begin{aligned}
G_{P}(x, \tau) & =-\frac{1}{2 \pi} \delta\left(x^{2}\right) \delta(\tau)-\frac{c_{5}}{2 \pi^{2}} \frac{\partial}{\partial x^{2}} \theta\left(-g_{55} g_{\alpha \beta} x^{\alpha} x^{\beta}\right) \frac{1}{\sqrt{-g_{55} g_{\alpha \beta} x^{\alpha} x^{\beta}}} \\
& =G_{\text {Maxwell }}+G_{\text {Correlation }}
\end{aligned}
$$

where $G_{\text {Maxwell }}$ breaks a higher symmetry to $O(3,1)$ while the support of $G_{\text {Correlation }}$ is

$$
-g_{55} g_{\alpha \beta} x^{\alpha} x^{\beta}= \begin{cases}-\left(x^{2}+c_{5}^{2} \tau^{2}\right)=c^{2} t^{2}-\mathbf{x}^{2}-c_{5}^{2} \tau^{2}>0, & g_{55}=1 \\ \left(x^{2}-c_{5}^{2} \tau^{2}\right)=\mathbf{x}^{2}-c^{2} t^{2}-c_{5}^{2} \tau^{2}>0 & , g_{55}=-1\end{cases}
$$

with causality properties dependent on the choice of $g_{55}$.

The contribution from $G_{\text {Correlation }}$ is smaller than that of $G_{\text {Maxwell }}$ by $c_{5} / c$ and drops off as $1 /|\mathbf{x}|^{2}$, so it may be neglected at low energy [21]. The contribution to the potential from $G_{\text {Maxwell }}$ is

$$
\begin{aligned}
a^{\alpha}(x, \tau) & =-\frac{e}{c} \int d^{4} x^{\prime} d \tau^{\prime} G_{\text {Maxwell }}\left(x-x^{\prime}, \tau-\tau^{\prime}\right) j_{\varphi}^{\alpha}\left(x^{\prime}, \tau^{\prime}\right) \\
& =\frac{e}{2 \pi c} \int d^{4} x^{\prime} d \tau^{\prime} d s \delta\left[\left(x-x^{\prime}\right)^{2}\right] \delta\left(\tau-\tau^{\prime}\right) \varphi\left(\tau^{\prime}-s\right) j^{\alpha}\left(x^{\prime}, s\right)
\end{aligned}
$$

and because $G_{\text {Maxwell }}$ has support at equal- $\tau$ this can be written

$$
a^{\alpha}(x, \tau)=\frac{e}{2 \pi c} \int d s \varphi(\tau-s) \int d^{4} x^{\prime} \delta\left[\left(x-x^{\prime}\right)^{2}\right] j^{\alpha}\left(x^{\prime}, s\right)
$$

expressing the potential as an ensemble of single-event potentials. Inserting the current defined in (20)

$$
j^{\alpha}(x, \tau)=c \dot{X}^{\alpha}(\tau) \delta^{4}(x-X(\tau))
$$


and using the identity

$$
\int d \tau f(\tau) \delta[g(\tau)]=\frac{f\left(\tau_{R}\right)}{\left|g^{\prime}\left(\tau_{R}\right)\right|}
$$

where $\tau_{R}$ is the retarded time that solves

$$
g(\tau)=\left(x-X\left(\tau_{R}\right)\right)^{2}=0 \quad \theta^{r e t}=\theta\left(x^{0}-X^{0}\left(\tau_{R}\right)\right),
$$

we find the potential

$$
\begin{aligned}
a^{\alpha}(x, \tau) & =\frac{e}{2 \pi} \int d s \varphi(\tau-s) \dot{X}^{\alpha}(s) \delta\left[\left(x-X^{\alpha}(s)\right)^{2}\right] \\
& =\frac{e}{4 \pi} \varphi\left(\tau-\tau_{R}\right) \frac{\dot{X}^{\alpha}\left(\tau_{R}\right)}{\left|\left(x^{\mu}-X^{\mu}\left(\tau_{R}\right)\right) \dot{X}_{\mu}\left(\tau_{R}\right)\right|}
\end{aligned}
$$

which is the standard Liénard-Wiechert potential multiplied by $\varphi\left(\tau-\tau_{R}\right)$. Thus, while the current that sources the pre-Maxwell field represents an ensemble of events along the worldline, the retarded causality of the Green's function selects the one member of the ensemble that intersects the lightcone of the observation point. The remaining $\tau$-dependence of the fields resides in the finite function $\varphi$ and expresses the relative time synchronization between the source and a test event experiencing the potential at the spacetime point $x$ at the chronological time $\tau$.

To find the Coulomb potential, we specify the event trajectory $X(\tau)=(c \tau, \mathbf{0})$ which produces the instantaneous current

$$
j^{0}(x, \tau)=c^{2} \delta(t-\tau) \delta^{3}(\mathbf{x}) \quad \mathbf{j}(x, \tau)=0 \quad j^{5}(x, \tau)=c c_{5} \delta(t-\tau) \delta^{3}(\mathbf{x})
$$

and so the potential

$$
a^{0}(x, \tau)=\frac{e}{4 \pi|\mathbf{x}|} \varphi\left(\tau-\left(t-\frac{|\mathbf{x}|}{c}\right)\right) \quad \mathbf{a}=0 \quad a^{5}(x, \tau)=\frac{c_{5}}{c} a^{0}(x, \tau)
$$

is found from from $G_{\text {Maxwell }}$. Consider a test event $x(\tau)=\left(c\left(\tau-\tau_{0}\right), \mathbf{x}\right)$ evolving along a parallel trajectory at spacial separation $\mathbf{x}$ and time offset $\tau_{0}$ so that

$$
\varphi\left(\tau-\left(t-\frac{|\mathbf{x}|}{c}\right)\right)=\frac{1}{2 \xi} e^{-\frac{1}{\xi \lambda}\left|\tau_{0}+\right| \mathbf{x}|/ c|} .
$$

If the test event is precisely on the forward lightcone of the source with $\tau_{0}=-|\mathbf{x}| / c$ then $\varphi=1$ and the interaction is purely Coulomb in form. If $\tau_{0}=0$ so that the events are synchronized at $x^{0}$, then

$$
a^{0}(x, \tau)=\frac{1}{2 \xi} \frac{e}{4 \pi|\mathbf{x}|} e^{-|\mathbf{x}| / \xi \lambda c}
$$

which has the form of a Yukawa-type potential with photon mass $m_{\gamma} \sim \hbar / \xi \lambda c^{2}$. If the source and test events are slightly desynchronized, with $\tau_{0}>0$, then the interaction is weakened by a factor $e^{-\tau_{0} / \xi \lambda c}$.

Thus, the factor $\lambda$ that characterizes the width of the ensemble and represents the average event inter-occurrence time, also determines the mass spectrum of the photons mediating the interaction between events. As proposed by Stueckelberg and seen in the second of (19), the masses of the particles and fields are not separately conserved, although Noether's theorem for affine $\tau$-displacement symmetry guarantees that the total mass is a constant of the motion [22]. If $\lambda$ is small (so that $\varphi / \lambda$ approaches a delta function and the current narrows to a small neighborhood around the event), the mass spectrum becomes wide and the interaction range and cross-section decreases. If $\lambda$ is large, the support of the current spreads along the worldline, the potential becomes Coulomb-like and the photon mass spectrum is small. 
A similar role is seen for $\lambda$ in SHP quantum field theory. From the Fourier expansion for the electromagnetic Green's function (43)

$$
G\left(x-x^{\prime}, \tau-\tau^{\prime}\right)=\int \frac{d^{4} k d \kappa}{(2 \pi)^{5}} \frac{e^{i\left[k \cdot\left(x-x^{\prime}\right)+g_{55} \kappa c_{5}\left(\tau-\tau^{\prime}\right)\right]}}{k^{2}+g_{55} \kappa^{2}-i \epsilon}
$$

it appears that photon loops in the 5D theory would render it non-renormalizable. However, quantization of the higher order field kinetic term leads to the photon propagator factor

$$
\left[g^{\mu \nu}-\frac{k^{\mu} k^{\nu}}{k^{2}}\right] \frac{-i}{k^{2}+g_{55} \kappa^{2}-i \epsilon} \frac{1}{1+(\lambda \xi)^{2} \kappa^{2}}
$$

and the theory is super-renormalizable at second order. Again, we notice that if $\lambda$ is large, then large values of photon mass $\kappa$ are suppressed.

While mass exchange must be present in any classical theory of pair processes and must also be small to account for standard electromagnetic phenomenology, such a compromise cannot explain the fixed masses of elementary particles. However, it has been shown that under certain circumstances [23] a self-interaction induced through $G_{\text {Correlation }}$ has the effect of restoring on-shell evolution in event trajectories and thus returning the particle worldline to the observed fixed mass. As seen in (45) when $g_{55}=1$, the Green's function has time-like support, permitting the event to interact with the field produced earlier along its worldline. The net effect of this self-interaction is a damping (or anti-damping) force that accelerates the event evolution to its asymptotic mass shell. A more general approach is found in the statistical mechanics of the many-event system. While the model presented here describes a particle as a weighted ensemble of events $\varphi(s) X^{\mu}(\tau-s)$ along a single worldline, Horwitz has modeled [24] a particle as an ensemble of $n$ independent spacetime events $X_{i}^{\mu}(\tau), i=1,2, \ldots, n$ defined at a given $\tau$. He has shown that the total particle mass is determined by a chemical potential. Following collisions governed by a general class of interactions that includes pair processes, particles return to their equilibrium mass values. These developments indicate that the statistical mechanics of event ensembles in the construction of classical particles will be a fruitful way to understand mass and perhaps derive masses from first principles.

\section{Maxwell Theory as an Equilibrium State of SHP}

Following an argument by Stueckelberg, Saad et al. [13] noticed that under the boundary conditions

$$
j_{\varphi}^{5}(x, \tau) \underset{\tau \rightarrow \pm \infty}{\longrightarrow} 0 \quad f^{5 \mu}(x, \tau) \underset{\tau \rightarrow \pm \infty}{\longrightarrow} 0
$$

integration of the pre-Maxwell equations provide

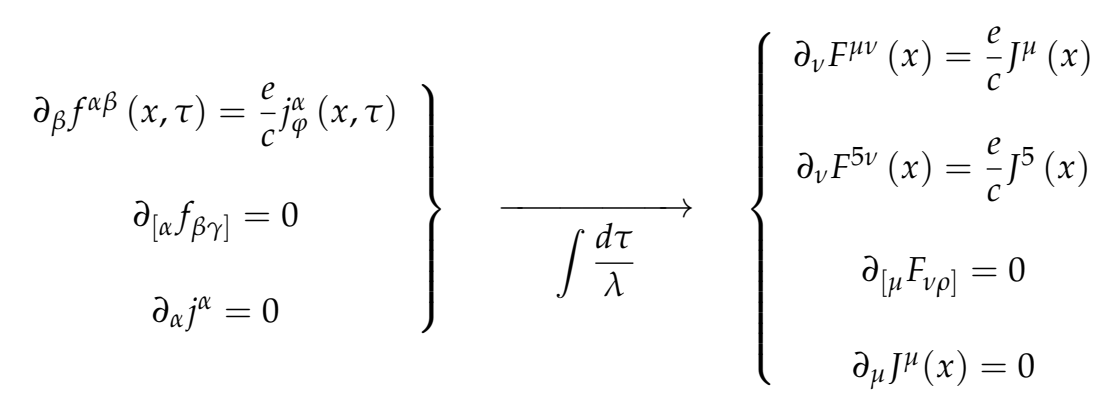

where

$$
A^{\alpha}(x)=\int \frac{d \tau}{\lambda} a^{\alpha}(x, \tau) \quad F^{\alpha v}(x)=\int \frac{d \tau}{\lambda} f^{\alpha v}(x, \tau) .
$$

This integration has been called concatenation and is understood as aggregation of all events that occur at a spacetime point $x$ over all $\tau$. The decoupling of $F^{5 \mu}$ from $F^{\mu v}$, which now satisfies Maxwell's 
equations, suggests that the SHP can be seen as an underlying microscopic dynamics for which Maxwell theory is an equilibrium or expectation state. In particular, we see that integration of (54) provides the Coulomb potential and using (27) to integrate the 4-vector current yields the Maxwell current $J^{\mu}(x)$ in the standard form as

$$
\begin{aligned}
\int d \tau j_{\varphi}^{\mu}(x, \tau) & =c \int d \tau d s \varphi(\tau-s) \dot{X}^{\mu}(s) \delta^{4}(x-X(s)) \\
& =c \int d \tau \dot{X}^{\mu}(\tau) \delta^{4}(x-X(\tau)) .
\end{aligned}
$$

Similarly, concatenation of (43)

$$
\int d \tau G_{\text {Maxwell }}=D(x)=-\frac{1}{2 \pi} \delta\left(x^{2}\right) \quad \int d \tau G_{\text {Correlation }}=0
$$

recovers the 4D Maxwell Green's function.

Another approach [21] to retrieving Maxwell theory from SHP is to slow the $\tau$-evolution to zero by taking $c_{5} / c \rightarrow 0$, thus freezing the microscopic system into a static equilibrium. Under this condition, the homogeneous Equation (30) imposes the condition

$$
c_{5}\left(\partial_{\nu} f_{5 \mu}-\partial_{\mu} f_{5 v}\right)+\partial_{\tau} f_{\mu \nu}=0 \underset{c_{5} \rightarrow 0}{\longrightarrow} \partial_{\tau} f_{\mu \nu}=0
$$

requiring that the field strength $f^{\mu v}$ be $\tau$-independent in this limit. As seen in the Liénard-Wiechert potential, the $\tau$-dependence resides in $\varphi\left(\tau-\tau_{R}\right)$ and can only be suppressed by taking $\lambda \rightarrow \infty$. We recall that $\lambda$ is the correlation time for the current $j^{\alpha}$, that is the measure of information about the interaction at time $\tau$ available from observation of the interaction at time $s<\tau$. Thus, the action-at-a-distance Coulomb law can be understood as the effect of a long-term correlation for the instantaneous potential. Applying the combined limits on $c_{5} / c$ and $\lambda$ to (25) and (26), we find $\varphi(\tau) \rightarrow 1 / 2 \xi=1$ and so all field components must be $\tau$-independent. This requirement effectively assigns equal weight to all event currents $j^{\alpha}(x, \tau)$ in the ensemble $j_{\varphi}^{\alpha}(x, \tau)$ defined along the worldline, recovering the standard particle current through

$$
\begin{aligned}
j_{\varphi}^{\mu}(x, \tau)=\int d s \varphi(\tau-s) j^{\mu}(x, s) & \longrightarrow \int d s 1 \cdot j^{\mu}(x, s)=J^{\mu}(x) \\
j_{\varphi}^{5}(x, \tau)=\int d s \varphi(\tau-s) j^{5}(x, s) & \longrightarrow \int d s j^{5}(x, s) \\
\partial_{\mu} j_{\varphi}^{\mu}(x, \tau)+\frac{1}{c_{5}} \partial_{\tau} j_{\varphi}^{5}(x, \tau)=0 & \longrightarrow \partial_{\mu} J^{\mu}(x)=0
\end{aligned}
$$

of Maxwell theory. Note that the current $\frac{1}{c_{5}} j_{\varphi}^{5}(x, \tau)$ remains finite because $j^{5}(x, \tau)$ includes the factor $\dot{X}^{5}=c_{5}$. As expected, $f^{\mu v}$ decouples from $f^{5 \mu}$ and satisfies Maxwell's equations, while the photon mass $m_{\gamma} \sim \hbar / \xi \lambda c^{2}$ vanishes.

\section{Conclusions}

Stueckelberg-Horwitz-Piron electrodynamics can be approached as an abstract gauge theory, exploring the consequences of allowing the gauge transformation (12) of the quantum wave function to depend on the evolution parameter in the dynamical framework. However, in another sense, just as Maxwell sought to formalize the empirical results of Cavendish and Coulomb, SHP may be seen as accounting for classical Maxwell electrodynamics in light of the pair creation/annihilation phenomena observed by Anderson. For Stueckelberg, pair processes provide empirical evidence that time must be understood as two distinct physical phenomena, chronology and coordinate, and so must be formalized through independent quantities $\tau$ and $\left(x^{0}, \mathbf{x}\right)$ in a physically reasonable theory. 
Having become accustomed, during the two hundred years that separate Cavendish from general gauge theory, to characterizing a single physical time by the evolution of specialized machines (clocks) in a coordinate frame, it is unsurprising that introducing such a distinction raises conceptual difficulties.

A new phenomenon in SHP is the absence of a static configuration-a particle may only remain at the origin in its rest frame for all coordinate time $x^{0}$ if its underlying microscopic event continually and uniformly evolves along its time axis, as $x=\left(c\left(\tau+\tau_{0}\right), \mathbf{0}\right)$. The coordinate $x^{0}=c \tau_{0}$ at $\tau=0$ is not simply an artifact of initializing a system clock, because the field induced by this event trajectory

$$
a^{0}(x, \tau)=\frac{e}{4 \pi|\mathbf{x}|} \varphi\left(\tau+\tau_{0}-\left(t-\frac{|\mathbf{x}|}{c}\right)\right) \quad a^{5}(x, \tau)=\frac{c_{5}}{c} a^{0}(x, \tau)
$$

depends explicitly on the constant $\tau_{0}$. The irreversible concatenation performed by measuring apparatus recovers the familiar Coulomb potential

$$
A^{0}(x)=\int \frac{d \tau}{\lambda} a^{0}(x, \tau)=\frac{e}{4 \pi|\mathbf{x}|}
$$

with no dependence on $\tau_{0}$ or even on the details of the weight function $\varphi(\tau)$. Similarly, $\tau_{0}$ plays no role in the quantized theory where sharply defined mass-momentum states retain no information about the initial conditions of coordinates. Nevertheless, in SHP, the microscopic event dynamics are determined by the Lorentz force (19) and so a test event at $x(\tau)=\left(c\left(\tau+\tau_{0}^{\prime}\right), \mathbf{x}\right)$ will experience the Coulomb force

$$
M \ddot{\mathbf{x}}=-e^{2} \frac{1-g_{55} \frac{c_{5}}{c}}{1+\left(\frac{c_{5}}{c}\right)^{2}} \nabla\left(\frac{e^{-\left|c\left(\tau_{0}-\tau_{0}^{\prime}\right)+\right| \mathbf{x}|| / \tilde{\lambda} \lambda \mathcal{c}}}{4 \pi|\mathbf{x}|}\right)
$$

depending on the synchronization $\tau_{0}-\tau_{0}^{\prime}$. Regarding (64) as a Yukawa potential, the limit $\lambda \rightarrow 0$ is understood as extending the range of the interaction to a Coulomb form by taking the mass of the photons that carry the interaction to zero. Viewing $\lambda$ as a correlation time for the microscopic current density $j^{\alpha}(x, \tau)$, this limit can be understood as smoothing the interaction to a time-independent Coulomb form by extending the correlation between values of the potential at different times along the entire worldline.

The structure of the source current for the pre-Maxwell field equations was seen to be determined by the choice of kinetic term for the fields. Standard field theory texts note that this choice is not imposed by physical foundations, but recommend the simplest form $f^{\alpha \beta} f_{\alpha \beta}$ because it is Lorentz and gauge invariant, contains only first order derivatives, and in the case of Maxwell theory, recovers the known field equations. For SHP, this choice is equivalent to taking $\varphi(\tau)=\lambda \delta(\tau)$, which does recover the concatenated Coulomb force through (65) but renders the Lorentz force

$$
M \ddot{\mathbf{x}}=-\lambda e^{2}\left(1-g_{55} \frac{c_{5}}{c}\right) \nabla\left[\frac{1}{4 \pi|\mathbf{x}|} \delta\left(\tau_{0}-\tau_{0}^{\prime}+|\mathbf{x}| / c\right)\right]
$$

difficult, if not impossible, to reconcile with known phenomenology. The smooth (but non-local) current was found by adding the higher-derivative term $\left(\partial_{\tau} f^{\alpha \beta}\right)\left(\partial_{\tau} f_{\alpha \beta}\right)$, which does not affect the Lorentz and gauge invariance of the action. A term of this type has also been considered by Pavsic for brane interactions [25].

Limits on the values of the parameters in SHP can be found from standard phenomenology. To describe elastic particle-antiparticle scattering, (66) undergoes

$$
-e^{2}\left(1-g_{55} \frac{c_{5}}{c}\right) \rightarrow e^{2}\left(1+g_{55} \frac{c_{5}}{c}\right)
$$

so that the experimental error in the asymmetry of scattering cross-sections places a limit on $c_{5} / c \ll 1$ and allows us to take $\xi \sim 1 / 2$. The width of the distribution $\varphi(\tau)$ is characterized by the parameter $\lambda$, which also determines as $m_{\gamma} \sim \hbar / \xi \lambda c^{2}$ the mass spectrum of the photons that mediate the induced 
force. Taking $m_{\gamma}$ to be the experimental error on the mass of the photon $\left(10^{-18} \mathrm{eV} / \mathrm{c}^{2}\right)$, we may estimate $\lambda>10^{-2}$ seconds. Thus, only very low energy interactions will produce phenomena that can be distinguished from standard Maxwell electrodynamics. Nevertheless, two distinct experimental signatures have been described. It was shown in [26] that the Liénard-Wiechert potential for a classical linear particle trajectory experiencing virtual particle-antiparticle processes will differ slightly from the potential predicted by Maxwell theory. In addition, it was shown in [15] that the scattering cross-section for scalar particles will differ slightly from the Klein-Nishina formula.

The smoothing of the single-event current by $\varphi(\tau)$ can be understood as constructing a statistical ensemble of events as the source for the field equations. An event on the trajectory $X^{\mu}(\tau)$ is associated with an ensemble whose members are of the form $\varphi(s) X^{\mu}(\tau-s)$, where the weight $\varphi(s)$ is the probability that a process generating independent random events at a constant average rate will produce an event occurring at displacement $s$ from time $\tau$. A single member of the ensemble is selected by the causal properties of the Green's function when determining the potential induced by the event trajectory. In this way, classical statistical mechanics is fundamental to the concept of a single-particle system and to the construction of a well-posed relativistic Hamiltonian theory of electromagnetism.

Acknowledgments: The author gratefully acknowledges the support of the Committee for the Encouragement of Research at Hadassah Academic College for covering the costs to publish in open access.

Conflicts of Interest: The author declares no conflict of interest.

\section{References}

1. Stueckelberg, E.C.G. La signification du temps propre en mécanique: Ondulatoire. Helv. Phys. Acta 1941, 14, 321-322. (In French)

2. Stueckelberg, E.C.G. Remarquea propos de la création de paires de particules en théorie de relativité. Helv. Phys. Acta 1941, 14, 588-594. (In French)

3. Horwitz, L.P.; Piron, C. Relativistic Dynamics. Helv. Phys. Acta 1973, 48, 316-326.

4. Horwitz, L.P.; Lavie, Y. Scattering theory in relativistic quantum mechanics. Phys. Rev. D 1982, 26, 819-838, doi:10.1103/PhysRevD.26.819.

5. Arshansky, R.I.; Horwitz, L.P. Relativistic potential scattering and phase shift analysis. J. Math. Phys. 1989, 30, 213, doi:10.1063/1.528572.

6. Arshansky, R.I.; Horwitz, L.P. Covariant phase shift analysis for relativistic potential scattering. Phys. Lett. A 1988, 131, 222-226.

7. Arshansky, R.I.; Horwitz, L.P. The quantum relativistic two-body bound state. I. The spectrum. J. Math. Phys. 1989, 30, 66, doi:10.1063/1.528591.

8. Arshansky, R.I.; Horwitz, L.P. The quantum relativistic two-body bound state. II. The induced representation of SL (2, C). J. Math. Phys. 1989, 30, 380, doi:10.1063/1.528456.

9. Land, M.C.; Arshansky, R.I.; Horwitz, L.P. Selection rules for dipole radiation from a relativistic bound state. Found. Phys. 1994, 24, 563-578, doi:10.1007/BF02058064.

10. Land, M.C.; Horwitz, L.P. The Zeeman effect for the relativistic bound state. J. Phys. A Math. Gen. 1995, 28, 3289-3304, doi:10.1088/0305-4470/28/11/025.

11. Land, M.C.; Horwitz, L.P. The Covariant Stark Effect. Found. Phys. 2001, 31, 967-991.

12. Seidewitz, E. Avoiding Haag's Theorem with Parameterized Quantum Field Theory. Found. Phys. 2017, 47, 355-374.

13. Saad, D.; Horwitz, L.P.; Arshansky, R.I. Off-shell electromagnetism in manifestly covariant relativistic quantum mechanics. Found. Phys. 1989, 19, 1125-1149.

14. Land, M.C.; Shnerb, N.; Horwitz, L.P. On Feynman's approach to the foundations of gauge theory. J. Math. Phys. 1995, 36, 3263, doi:10.1063/1.531030.

15. Land, M.C.; Horwitz, L.P. Offshell quantum electrodynamics. J. Phys. Conf. Ser. 2013, 437, 012011, doi:10.1088/1742-6596/437/1/012011.

16. Feynman, R.P. Mathematical formulation of the quantum theory of electromagnetic interaction. Phys. Rev. $1950,80,440-457$.

17. Feynman, R.P. Space-time approach to nonrelativistic quantum mechanics. Rev. Mod. Phys. 1948, 20, 367-387. 
18. Horwitz, L.P.; Arshansky, R.I.; Elitzur, A.C. On the two aspects of time: The distinction and its implications. Found. Phys. 1988, 18, 1159-1193.

19. Jackson, J.D. Classical Electrodynamics; Wiley: New York, NY, USA, 1975; p. 601.

20. Land, M.C.; Horwitz, L.P. Green's functions for off-shell electromagnetism and spacelike correlations. Found. Phys. 1991, 21, 299-310.

21. Land, M.C. Speeds of light in Stueckelberg-Horwitz-Piron electrodynamics. In Proceedings of the International Association for Relativistic Dynamics 2016 (IARD 2016), Ljubljana, Slovenia, 6-9 June 2016.

22. Land, M.C.; Horwitz, L.P. The Lorentz Force and Energy-Momentum for Off-Shell Electromagnetism. Found. Phys. Lett. 1991, 4, 61.

23. Land, M.C. Mass stability in classical Stueckelberg-Horwitz-Piron electrodynamics. In Proceedings of the International Association for Relativistic Dynamics 2016 (IARD 2016), Ljubljana, Slovenia, 6-9 June 2016.

24. Horwitz, L.P. A Statistical Mechanical Model for Mass Stability in the SHP Theory. arXiv 2016, arXiv:1607.03742v2.

25. Pavsic, M. Branes and Quantized Fields. Relativistic Membranes and Quantized Fields. In Proceedings of the International Association for Relativistic Dynamics 2016 (IARD 2016), Ljubljana, Slovenia, 6-9 June 2016.

26. Land, M.C.; Horwitz, L.P. Field signature for apparently superluminal particle motion. J. Phys. Conf. Ser. 2015, 615, 012008, doi:10.1088/1742-6596/615/1/012008.

(C) 2017 by the author. Licensee MDPI, Basel, Switzerland. This article is an open access article distributed under the terms and conditions of the Creative Commons Attribution (CC BY) license (http://creativecommons.org/licenses/by/4.0/). 